\title{
Neue \\ Datenschutzstrukturen in Europa?
}
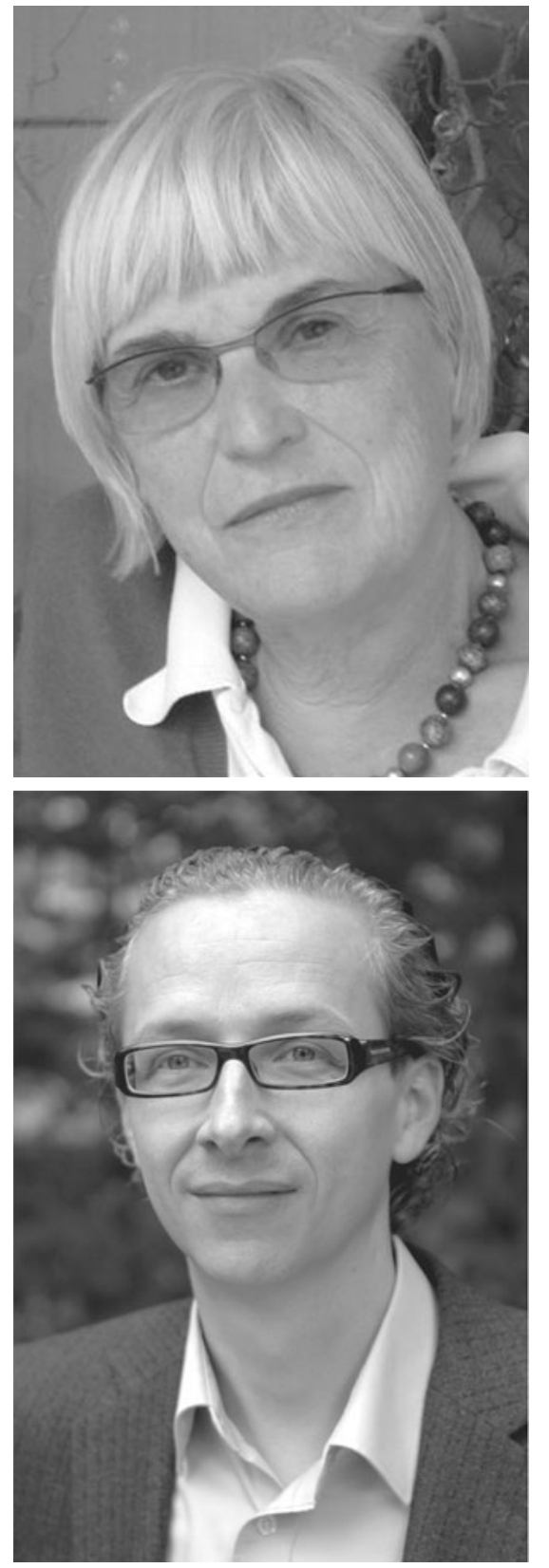

Die gewaltigen und beispiellosen Veränderungen der weltweiten Kommunikationsprozesse und ihrer Bedeutung vor Ort verlangen neue Erklärungs- und Deutungsansätze, neue Schlüssel auch durch das Recht. Das bedeutet keine Entwertung der hier in Rede stehenden grundrechtlichen Werte von Datenschutz und Informationsfreiheit. Ihre Deutungsmuster und gesetzlichen Ausprägungen sind aber unter internationalen und unionsrechtlichen sowie unter Aspekten rechtskultureller Grenzen nicht nur in den europäischen Staaten zu sehen.

Das Heft beginnt mit den Ausführungen der polnischen Bürgerbeauftragten und Verfassungsrechtlerin Irena Lipowicz zum grundrechtlich angreifbaren Anti-Counterfeiting Trade Agreement (ACTA), welches Ende Januar von Vertretern der EUKommission sowie von 22 Mitgliedstaaten der Union in Tokio unterzeichnet wurde. Die Verfasserin äußert unter den Aspekten Datenschutz, Informationsfreiheit und faires Verfahren erhebliche Bedenken gegenüber dem Abkommen. Vor kurzem hat die Kommission beschlossen, das Vertragswerk vom EuGH auf seine Vereinbarkeit mit europäischen Grundrechten überprüfen zu lassen; es bleibt zu hoffen, dass die in dem Beitrag angesprochenen Grundrechtsdefizite vom Gerichtshof entsprechend berücksichtigt werden.

Die Debatte um die geplante Datenschutzgrundverordnung der Union ist mittlerweile in vollem Gange. Der Entwurf strebt sicherlich notwendige datenschutzrechtliche Neuerungen an, etwa für die Datenverarbeitung im Online-Bereich. Andererseits zielt er in seiner derzeitigen Fassung auch darauf ab, nationale Mitgestaltungsrechte zurückzudrängen bzw. aufzuheben. Mit dieser Problematik setzen sich Hans-Hermann Schild und Marie-Theres Tinnefeld auseinander. Der Beitrag von Alexander Dix befasst sich ebenfalls mit den Vorschlägen der Europäischen Kommission. Der Autor spricht das zentrale Dilemma bei der Harmonisierung des europäischen Datenschutzrechts an, wonach die Aufsichtsbehörden in den Mitgliedstaaten zwar die Anwendung der Datenschutzgrundverordnung überwachen sollen, die Kommission sich aber in diesem Verfahren gleichzeitig Befugnisse beimisst, die über das zulässige Maß hinausgehen. Auch für das in Deutschland immer noch in der Schwebe befindliche Beschäftigtendatenschutzgesetz wird der Kommissionsentwurf von Relevanz sein. Martin Franzen beschäftigt sich aus diesem Anlass in seinem Beitrag mit den Auswirkungen des Unionsrechts auf das nationale (Arbeitnehmerdatenschutz-)Recht. Der Arbeitnehmerdatenschutz ist auch Thema der Ausführungen von Tsubasa Wakabayashi. Die Autorin gibt am Beispiel des japanischen Datenschutzrechts einen Einblick in eine ganz andere Art von (Rechts-)Kultur und thematisiert das Verhältnis zwischen Datenschutz und Diskriminierungsschutz.

Zweiter Themenschwerpunkt des Hefts ist das Spannungsverhältnis zwischen Datenschutz und Sicherheit. Phillip Brunst nimmt sich dieses Themas am Beispiel staatlicher (Anti-)Krypto-Strategien an, wobei er uns anschaulich das „Krypto-Dilemma" vor Augen führt, dass Kryptographie gleichermaßen gefährlich (Kommunikation von Straftätern und Terroristen) wie wichtig (Kommunikation von Bür- 


\section{Hier steht eine Anzeige.}

黛 Springer 
gerrechtlern, E-Business) ist. Franziska Boehm setzt sich mit dem Problem der immer weiter ausufernden Datenzugriffe von europäischen Strafverfolgungsbehörden auseinander und macht wenig Hoffnung, dass sich dies bei Umsetzung der Kommissionsvorschläge für eine neue Richtlinie zum Datenschutz im Bereich Polizei und Justiz ändern wird.

Der Traum von EU-Projekten wie INDECT ist der intelligente Einsatz von Technik zur Verbrechensprävention, menschliche Bewegungs- und Verhaltensmuster sollen ausgewertet werden, um mögliche Gewaltbereitschaft oder andere vermeintliche Bedrohungen frühzeitig zu erkennen. Nochmals einen Schritt weiter gehen diejenigen, die sich vom Neuroimaging sogar die Fähigkeit zum Gedankenlesen erhoffen. Stephan Schleim plädiert in seinem Beitrag zu den Möglichkeiten und Grenzen der Neurowissenschaften für mehr Sachlichkeit und gegen falsche Hoffnungen. Er befasst sich vor allem mit fMRT/fMRI (Hirnscanner) als einer wichtigen Methode zur Untersuchung der menschlichen Gehirnfunktionen, die auch in Ermittlungsverfahren diskutiert und bereits eingesetzt wird. Rolf Sachsse und Hartmut Wagner schließlich gehen auf die besondere "Macht der Bilder" ein und warnen vor einer "Koalition der technologisch und ökonomisch besetzten, prozessierenden Bilderwelt mit einem technokratisch herrschaftsapotheotischen Verfassungsverständnis".

Yolande Stolte behandelt in ihrem Beitrag Freiheit und Grenzen journalistischer Recherche, die in England nicht nur wegen WikiLeaks, sondern vor allem auch infolge des "News of the World"-Abhörskandals besonders kontrovers diskutiert werden. Olena Prodan rundet das Heft mit ihrem Beitrag zum neuen Datenschutzrecht der Ukraine ab und beleuchtet die von der Ukraine vorangetriebene Angleichung des nationalen Rechts an das europäische Datenschutzrecht.

\section{Marie-Theres Tinnefeld, Benedikt Buchner}

